\title{
Applications of Metabolomics to Precision Nutrition
}

\author{
Marcia LeVatte ${ }^{a} \quad$ Ammar Hassanzadeh Keshteli $^{a} \quad$ Parvin Zarei $^{b}$ \\ David S. Wisharta, ${ }^{\mathrm{a}, \mathrm{d}} \mathrm{d}, \mathrm{e}$
}

aDepartment of Biological Sciences, University of Alberta, Edmonton, AB, Canada; ${ }^{b}$ Department of Bioinformatics, Isfahan University of Medical Sciences, Isfahan, Iran; 'Department of Computing Sciences, University of Alberta, Edmonton, AB, Canada; 'Department of Laboratory Medicine and Pathology, University of Alberta, Edmonton, AB, Canada; ${ }^{\mathrm{e}}$ Faculty of Pharmacy and Pharmaceutical Sciences, University of Alberta, Edmonton, AB, Canada

\section{Keywords}

Metabolomics · Lifestyle interventions - Biomarkers of health · Precision nutrition

\begin{abstract}
Background: For thousands of years, disabilities due to nutrient deficiencies have plagued humanity. Rickets, scurvy, anemia, stunted growth, blindness, and mental handicaps due to nutrient deficiencies affected up to 1/10 of the world's population prior to 1900 . The discovery of essential amino acids, vitamins, and minerals, in the early 1900s, led to a fundamental change in our understanding of food and a revolution in human health. Widespread vitamin and mineral supplementation, the development of recommended dietary allowances, and the implementation of food labeling and testing along with significant improvements in food production and food quality have meant that nutrient-related disorders have almost vanished in the developed world. The success of nutritional science in preventing disease at a population-wide level is one of the great scientific triumphs of the 20th century. The challenge for nutritional science in the 21 st century is to understand how to use nutrients and other food constituents to enhance human health or prevent dis-
\end{abstract}

karger@karger.com www.karger.com/lfg

Karger!"
C 2021 The Author(s).

Published by S. Karger AG, Basel

This is an Open Access article licensed under the Creative Common Attribution-NonCommercial-4.0 International License (CC BY-NC) (http://www.karger.com/Services/OpenAccessLicense), applicable to the online version of the article only. Usage and distribution for commercial purposes requires written permission. ease at a more personal level. This is the primary goal of precision nutrition. Summary: Precision nutrition is an emerging branch of nutrition science that aims to use modern omics technologies (genomics, proteomics, and metabolomics) to assess an individual's response to specific foods or dietary patterns and thereby determine the most effective diet or lifestyle interventions to prevent or treat specific diseases in that individual. Metabolomics is vital to nearly every aspect of precision nutrition. It can be used to comprehensively characterize the thousands of chemicals in foods, to identify food byproducts in human biofluids or tissues, to characterize nutrient deficiencies or excesses, to monitor biochemical responses to dietary interventions, to track long-term or short-term dietary habits, and to guide the development of nutritional therapies. In this review, we will describe how metabolomics has been used to advance the field of precision nutrition by providing some notable examples or use cases. First, we will describe how metabolomics helped launch the field of precision nutrition through the diagnosis and dietary therapy of individuals with inborn errors of metabolism. Next, we will describe how metabolomics is being used to comprehensively characterize the full chemical complexity of many key foods, and how this is revealing much more about nutrients than ever imagined.
Correspondence to:

David S.Wishart, dwishart@ualberta.ca 
Third, we will describe how metabolomics is being used to identify food consumption biomarkers and how this opens the door to a more objective and quantitative assessments of an individual's diet and their response to certain foods. Finally, we will describe how metabolomics is being coupled with other omics technologies to develop custom diets and lifestyle interventions that are leading to positive health benefits. Key Message: Metabolomics is vital to the advancement of nutritional science and in making the dream of precision nutrition a reality.

(c) 2021 The Author(s)

Published by S. Karger AG, Basel

\section{Introduction}

Optimal nutrition promotes prolonged good health, while poor nutrition contributes to chronic diseases such as cardiovascular disease, diabetes, obesity, or diseases of undernutrition such as maramus and kwashiorkor. Many developed countries have implemented nationwide mandatory mineral or vitamin supplementation along with population-wide recommended dietary allowances (RDAs) of certain foods (fruits and vegetables, dairy, and meat). These RDAs are set at a level that meets the needs for essential vitamins, minerals, amino acids, and fatty acids for maintaining good health in $97.5 \%$ of a specific population. Mandated micronutrient supplementation and nationwide RDAs have helped to significantly improve population-wide health in many countries. While RDAs are set as a goal for usual intake at the individual level, not everyone benefits from these RDAs in the same way. Indeed, there can be significantly different interindividual (cross-sectional differences between individuals) or intraindividual (longitudinal differences within a single individual) variations or responses to the same foods or the same nutrients [1]. These dietary responses are affected by age, genetics, gut microbiota, metabolism, physiology, physical activity, and lifestyle [2]. It is this realization of nutritional "individualism" that has led to the push toward precision nutrition.

Precision nutrition is a branch of nutrition science that aims to use genomics, proteomics, and/or metabolomics to assess an individual's response to specific foods or dietary patterns. By assessing these responses at a comprehensive, molecular level, it is possible to determine the most effective diet or lifestyle interventions to improve health or prevent and even treat specific diseases [3]. A key focus of both precision nutrition and precision medicine is on proactive intervention and prevention rather than reactive salvaging or saving. Catching a problem ear- ly and working to fix it usually prevents long-term or irreparable damage. Given its potential impact, the National Institutes of Health has promoted precision nutrition as the best strategy to develop clinically relevant and meaningful diet solutions for individuals and populations that share common physiological, behavioral, or sociocultural characteristics [4].

The emergence of precision nutrition has largely been tied to the emergence of sufficiently fast, cheap, and comprehensive genomics, proteomics, and metabolomics technologies. It is through these technologies that the necessary molecular measurements to precisely assess food composition, identify individual genetic propensities, or evaluate individual responses to diet and lifestyle interventions have become available. While most reviews on precision nutrition have focused on the use of genomics or transcriptomics technologies to advance the field $[5,6]$, in this review we shall focus on the role of metabolomics in precision nutrition. As we will show, metabolomics is vital to nearly every aspect of precision nutrition. Metabolomics is a field of omics science that uses cuttingedge analytical chemistry techniques and advanced computational methods to characterize complex biochemical mixtures [7]. It can be used to comprehensively characterize the thousands of chemicals in foods [8], to identify food byproducts in human biofluids or tissues [9], to characterize nutrient deficiencies or excesses $[10,11]$, to monitor biochemical responses to dietary interventions [12], to track long-term or short-term dietary habits [13], and to guide the development of nutritional therapies [14].

In this review, we will briefly introduce metabolomics and describe how it has become a key technology for nutritional science. We will then show how metabolomics has been used to advance the field of precision nutrition by providing some notable examples or case studies. First, we will describe how metabolomics played a key role in launching the field of precision nutrition through the diagnosis and dietary therapy of individuals with inborn errors of metabolism (IEMs). Next, we will describe how metabolomics can be used to comprehensively characterize the full chemical complexity of many key foods and how this is adding new meaning to the concept of molecular nutrition. Third, we will describe how metabolomics is being used to identify biomarkers of food intake (BFIs), and how this could open the door to a more objective and quantitative assessments of an individual's diet and their response to dietary changes. Finally, we will describe how metabolomics is being coupled with other omics technologies to develop custom diets and lifestyle 
interventions that are leading to positive health benefits for several chronic diseases. This review will conclude with a brief discussion regarding the future of metabolomics in precision nutrition.

\section{Metabolomics and Nutritional Science}

Metabolomics is the science of studying the metabolome. It is a branch of analytical chemistry that focuses on characterizing small molecules (molecular weights of $<1,500 \mathrm{Da}$ ) found in tissues, cells, or biofluids. Unlike genomics, transcriptomics, or proteomics, in which a single instrument is often sufficient to perform the necessary measurements, metabolomics requires a broad array of instrumentation. Over the past 15 years, 3 main technologies have emerged as the primary workhorses in metabolomics: nuclear magnetic resonance (NMR) spectroscopy [15], gas chromatography-mass spectrometry (GC-MS) [16], and liquid chromatography MS (LC-MS) $[17,18]$. Each technique provides broad coverage of many classes of organic compounds, including lipids, amino acids, sugars, biogenic amines, and organic acids. As a general rule, NMR is best at identifying and quantifying high abundance metabolites, while GC-MS and LC-MS are best at detecting lower abundance metabolites (shown in Fig. 1). Although these technologies each have their own advantages and disadvantages, numerous studies have shown how they may be used to complement each other [17, 19]. Indeed, the use of multiple technologies greatly broadens the level of metabolite coverage that can be achieved and the types of samples that can be studied.

Metabolomic approaches can either be targeted or untargeted. With targeted metabolomics, selected metabolites are identified and quantified by comparison to known chemical standards to help develop biomarkers or test hypotheses. With untargeted metabolomics, there is less focus on compound identification and quantification but more emphasis on novel compound discovery and discovery-based research [19]. Both untargeted and targeted metabolomics have their advantages and disadvantages, but given the importance attached to bioactive compound identification and quantification, there is a growing preference for quantitative metabolomics in many areas of food science and nutrition research. Indeed, targeted, quantitative metabolomics is now widely used in food composition analysis $[8,20,21]$, the identification of BFIs $[13,22]$, the detection and monitoring of nutritional deficiencies or metabolic/nutrient disorders

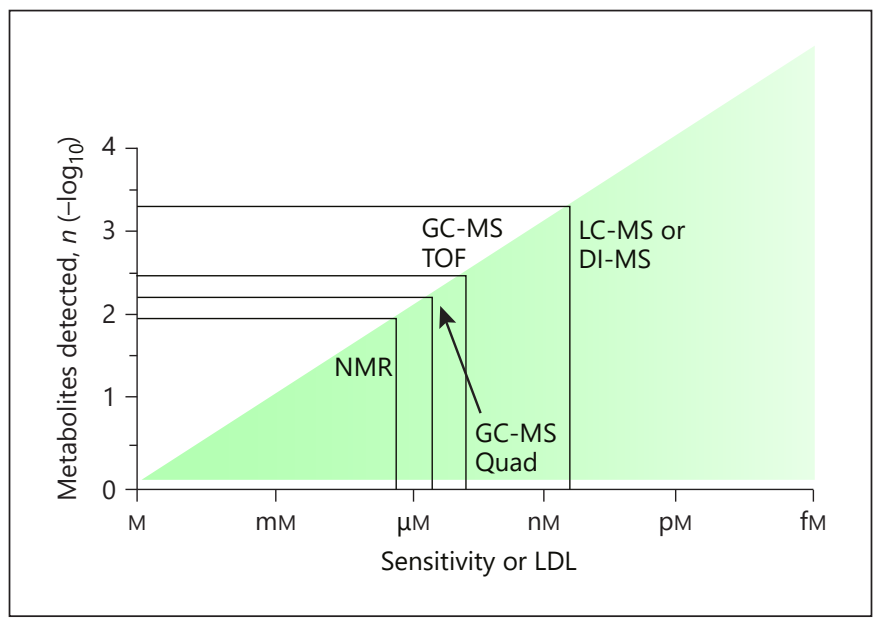

Fig. 1. Comparison of relative sensitivity or LDLs of the commonly used metabolomic platforms including NMR, GC-MS, and liquid chromatography LC-MS. LDLs, lower detection limits; NMR, nuclear magnetic resonance; GC-MS, gas chromatography-mass spectrometry; LC-MS, liquid chromatography-mass spectrometry.

$[10,11,23]$, the estimation of dietary intake [24-26], and the development of dietary recommendations for wellness and chronic disease prevention [14].

\section{Precision Nutrition and IEMs}

Perhaps, the most successful example of precision nutrition, to date, has been the application of targeted metabolomic methods toward newborn screening. Nearly every newborn child in the developed world is subject to a dried blood spot test that uses MS. These MS-based metabolomic tests can measure up to 40 different metabolites, including amino acids, organic acids, and acylcarnitines [27]. These metabolomic assays permit the diagnosis of up to 30 different metabolic disorders or IEMs [28]. Newborn screening is far and away the most successful example of any omics technology making its way to the clinic. Based on national testing data reported by many countries, we estimate that over the past 25 years $>400$ million children have been subject to newborn screening and up to 1 million have had their lives improved through early detection and intervention. In many cases, IEMs detected by MS-based newborn screening can be treated through customized dietary interventions that either supplement or eliminate a certain nutrient that causes the health effects arising from the IEM. Not only does MS- 
based newborn screening help in diagnosing or even predicting disease but the same metabolomic techniques can also be used to determine the optimal nutritional therapy, to monitor each individual's response, and to customize the composition of the medical food to suit the patient.

There are a number of examples with metabolomics and metabolite-guided dietary therapy succeeding in improving the lives of IEM patients. For instance, precision nutrition has long been used to treat patients with maple syrup urine disease (MSUD). MSUD is an autosomal recessive IEM that is usually first detected via MS-based newborn screening for high blood levels of branchedchain amino acids (BCAAs). MSUD affects an individual's ability to break down BCAAs such as leucine, isoleucine, and valine [29]. As a result, these BCAAs and their toxic byproducts ( $\alpha$-keto acids) buildup in the body. If untreated, MSUD can cause convulsions and permanent brain damage [30]. To prevent these BCAAs and keto acids from accumulating, MSUD patients are given specially developed medical foods that have been formulated to be free of leucine, isoleucine, and valine. These custom foods or beverage mixes contain all the amino acids (except the BCAAs) and appropriate protein content, vitamins, minerals, omega 3-polyunsaturated fatty acids, carbohydrates, and other fats needed for proper growth and development of affected individuals. Metabolomics is not only used to diagnose MSUD and guide the nutrient intervention but it is also used throughout an MSUD patient's life as each patient must test their urine daily for the buildup of keto acids using an at-home metabolomic colorimetric test, such as the dinitrophenyhydrazine assay, a nonquantitative screening test for keto acids [29].

Precision nutrition, guided by metabolomics, is also used to treat individuals with phenylketonuria (PKU). PKU is an autosomal recessive IEM that is usually first detected via MS-based newborn screening for high blood levels of phenylalanine. PKU affects a patient's ability to break down the amino acid phenylalanine [31]. The excess phenylalanine is converted to toxic metabolites (phenylketones) which accumulate in the blood and brain. If untreated, PKU can cause irreversible brain damage, seizures, and behavioral, emotional, and social problems [32]. To prevent the buildup of phenylketones, PKU patients must adhere to medical diets that are low in phenylalanine. Furthermore, they must avoid foods containing the artificial sweetener aspartame (which is converted to phenylalanine upon digestion). As those with PKU will not receive all the essential nutrients from their restricted diet, they must drink age-specific medical beverages free of phenylalanine that includes all other amino acids, vita- mins, minerals, carbohydrates, and fats to meet all their energy requirements and to support proper growth and development of affected individuals. PKU patients are regularly monitored via metabolomic methods for the buildup of phenylalanine and phenylketones through monthly blood tests.

Yet another example of an IEM that can be treated via metabolomics-guided precision nutrition is biotinidase deficiency (BTD). BTD is an autosomal recessive IEM that can be detected via MS-based newborn screening for low biotin levels. BTD prevents the vitamin biotin from being recycled by the body [33]. Biotin helps with the breakdown of protein, fats, and carbohydrates. If left untreated, those with BTD can develop breathing and balance problems, vision and hearing loss, and alopecia and skin rashes. BTD patients can avoid these neurological and dermatological disorders through oral biotin supplements [34]. Blood tests are not usually performed to monitor BTD patients; however, periodic monitoring for dietary noncompliance may be performed with younger individuals. Thus, for IEMs, metabolomics offers precise, personalized diagnoses that help catch the condition early so that precise diets, customized lifestyle changes, or individualized medical foods can be developed or prescribed. Indeed, metabolomics has been enabling precision nutrition for the proactive treatment of IEMs for $>2$ decades.

\section{Metabolomics and Comprehensive Food Characterization}

Understanding what nutrients and micronutrients are in our food is key to understanding their health-promoting or health-harming properties. This kind of molecular understanding is also key to the main goals of precision nutrition. Traditionally most food analyses have focused on measuring about 30-40 macronutrient or essential nutrients. These measurements are normally performed by national food agencies such as the USDA or Health Canada. Most developed countries have national food composition databases that provide detailed measurements of these nutrients for thousands of raw or prepared foods.

However, most foods actually contain thousands of compounds, not dozens [35]. Indeed, the average fruit or vegetable is estimated to contain $>15,000$ different compounds from $>100$ chemical classes with concentrations ranging from femtomolar (e.g., vitamins) to millimolar (e.g., sugars) [35]. Many of the unmeasured or less mea- 
Fig. 2. Standard food metabolomics workflow showing how samples are extracted and liquified and then analyzed via NMR, GC-MS, or LC-MS methods. Compound identification and quantification are often done through sophisticated annotation software. NMR, nuclear magnetic resonance; GC-MS, gas chromatography-mass spectrometry; LC-MS, liquid chromatography-mass spectrometry.

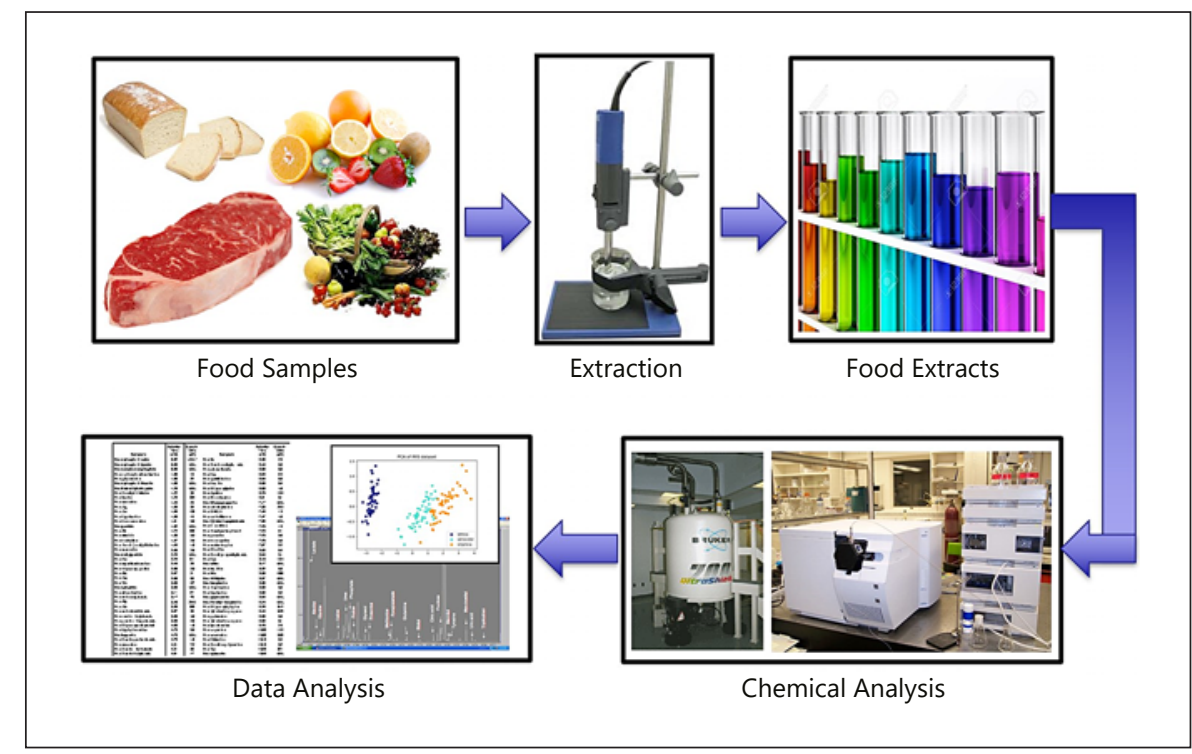

sured compounds in foods include micronutrients such as polyphenols, which have effects that could lead to important health benefits [36], terpenes which give many foods their flavor and aroma [37], and various pigments which give foods their color. The only way to detect, identify, and quantify this rich chemical diversity in foods is through metabolomics. A diagram illustrating standard metabolomic workflow for analyzing food samples is shown in Figure 2. Comprehensive MS and NMR metabolomics studies on milk [17], beef [38], bananas [39], wine [40], beer [41], rice [42], and tomatoes [43] have led to the identification of hundreds to thousands of previously undocumented chemicals in these foods and beverages. This work has led to the creation of a number of online food constituent or food metabolome databases such as Phenol-Explorer [44], PhytoHub [45], and FooDB [46]. Phenol-Explorer contains detailed data on 501 polyphenols from 459 different foods along with data on the effects of food processing on polyphenol contents on 161 polyphenols from 155 foods. PhytoHub includes data on $>1,800$ food phytochemicals (including many terpenes) and their metabolites from 356 different plant foods. FooDB, which is the largest of these databases, has information on $>71,000$ chemicals in nearly 800 different raw or lightly processed foods. FooDB is particularly notable for including information on the health benefits, flavor, color, and aroma characteristics for many of these food compounds. These food metabolome databases, which primarily capture metabolomics-derived food composition data, are becoming key resources for guiding preci- sion nutrition and informing nutrition scientists. This is because they provide rich, actionable information about the natural, unnatural, and even the unexpected compounds that can be found in common foods. This information can be used to formulate foods for medical purposes and advance our understanding of foods that have the desired ingredients at the desired concentrations to maximize health benefits.

\section{Metabolomics and BFIs}

For precision nutrition to deliver on its promises, it is vital to have a detailed understanding of an individual's diet and their overall nutritional status. Nutritional assessment has been traditionally done through surveys such as 24-h dietary recalls, food frequency questionnaires, or dietary diaries. However, these methods have many inherent errors and limitations. Problems with recall bias, subjectivity, deliberate deception, memory lapses, inability to estimate portion sizes, lack of preparation details, and food processing information can often lead to incorrect or incongruous data. Due to the limitations of these methods, there has been a strong push to use more objective analytical methods that are more precise and more reliable to measure an individual's food consumption. This led to a major initiative launched in 2013 called the Food Biomarker Alliance (FoodBAll). The goal of FoodBAll was to use metabolomics to identify biomarkers of food intake (BFIs) [47]. FoodBAll has created an 
inventory of metabolite markers in blood, urine, or other tissues/biofluids that can be used to reliably identify what a person has eaten and how (rapidly or slowly) that food has been metabolized. Understanding exactly what a person has eaten, how much they have eaten, and how it has been metabolized is critical to doing precision nutrition. Indeed, without this kind of reliable information, the development of personalized dietary interventions or the measurement of personalized dietary responses is almost impossible.

To catalog these BFIs, members of the FoodBAll consortium systematically reviewed the literature and conducted independent metabolomic studies to identify dozens of BFIs for many classes of foods [17, 48-52]. The FoodBAll consortium also developed protocols and definitions for BFI identification and validation [53, 54]. Many of these BFIs are now listed in the HMDB [55], MarkerDB [56], and in Exposome-Explorer [24]. Some of the more useful BFIs identified include urinary 1-methylhistidine and 3-methylhistidine as BFIs of meat intake [57], urinary proline-betaine as a BFI of citrus intake [58], urinary TMAO as a BFI of fish consumption [59], serum daidzein as a marker of soy intake $[60,61]$ and urinary 2 -furoylglycine as a BFI for coffee consumption [62]. In many cases, the performance of these biomarkers in terms of their sensitivity, specificity, and area under the receiver operating characteristic curve is quite impressive. In every case, these BFIs must be measured using targeted metabolomic methods.

BFIs offer nutrition scientists an unbiased, scientifically rigorous approach to molecularly assessing food consumption and avoiding the failings of food questionnaires. They also open the door to understanding individual differences in terms of dietary habits, food metabolism, and gut microbial activity. This kind of information is critical to implementing precision nutrition strategies. Indeed, without a solid understanding of what a person consumes and how their body processes, it is almost impossible to develop effective dietary or lifestyle interventions that could improve a person's health or well-being [63].

\section{Metabolomics and Precision Nutrition for Chronic Disease}

Metabolomics is now being used to understand the diet-related mechanisms associated with many chronic conditions such as cardiovascular disease, diabetes, and obesity [64-66]. Among the most impressive examples of how metabolomics and precision nutrition have complemented each other has been in diabetes control or prevention. Diabetes is rapidly becoming one of the most common life-threatening conditions in the developed world. Approximately $10 \%$ of adults in the United States are diabetic and 35\% are prediabetic [67]. To explore how metabolomics, metagenomics, and precision nutrition could work to control diabetes, Zeevi et al. [68] integrated these omics techniques with machine learning to develop customized dietary recommendations for controlling elevated postprandial blood glucose. In performing this study, the authors collected metabolomic-based blood parameters, anthropometric data, physical activity data, self-reported dietary intake, and gut microbial composition and function from 800 healthy and prediabetic participants. Participants were given standardized meals (glucose, bread, bread and butter, bread and chocolate, and fructose), and their blood glucose levels were continuously monitored for 1 week. Even though all participants ate the same food, they found a high interpersonal variability in postprandial glycemic responses. Using the data collected from these 800 individuals, they developed a machinelearning algorithm to accurately predict glycemic responses for each participant (based on their omic profile) after consuming different types of food. This algorithm was later successfully validated in another cohort of 100 individuals. This work led to the establishment of one of the first precision nutrition companies, DayTwo Inc., which specializes in using both individual metabolomics and microbial genomics data to create custom diets that can control or prevent prediabetes [69].

In another example of metabolomics being used to guide precision nutrition, Anwar et al. [70] conducted a study on 40 healthy adults with normal blood glucose levels to predict the risk of developing type 2 diabetes. At the beginning of the study, blood samples were collected from participants to quantify metabolite levels using LCMS-based metabolomics. The blood metabolite levels were then used to calculate health risk scores for the development of type 2 diabetes, insulin resistance, and associated comorbidities. Based on the analysis of their health risks scores, participants were provided with health reports with several lifestyle recommendations including personalized diets (guided by their dietary preferences and food allergies), exercise (based on physical limitations), and nutritional supplements to normalize their blood metabolite levels. After following the recommendations for 100 days, a follow-up metabolomic assessment showed significant reductions in the health risks associated with type 2 diabetes and associated comorbidi- 
ties. Larger studies of up to 1,000 North American adults showed similar positive results when participants were given personalized nutrition and lifestyle recommendations based on baseline dietary behavior, preferences, and health markers measured via metabolomics [71]. While most metabolomics-driven precision nutrition studies have been limited to controlling diabetes, prediabetes, and/or obesity, it is clear that these approaches are having positive effects. Given that diabetes, prediabetes, and/or obesity affect $>30 \%$ of American adults and given that they also lead to heightened risks for heart disease, stroke, and cancer, these success stories for precision nutrition and metabolomics are highly significant.

\section{Conclusion and Future Directions}

The application of metabolomics to diverse areas of nutritional and food science research has significantly extended our knowledge about the many chemicals in food. This work has also revealed the diversity of human metabolic responses to these chemicals. As highlighted in this review, the development of specialized food composition databases and the identification of robust BFIs using metabolomic platforms have provided new opportunities for researchers to design evidence-based dietary recommendations and to assess their effectiveness on human health. While the use of metabolomics to guide precision nutrition has largely been limited to treating IEMs and diabetes, the success of these metabolomics-informed interventions suggests that similar kinds of lifestyle interventions could be used to treat or prevent many other chronic conditions.

Certainly, there are a number of limitations and challenges to using metabolomics in nutritional research (extensively discussed by Maruvada et al. [72]). However, increased adoption is happening and important advances are being made. For instance, recent improvements in NMR and MS-based technologies for targeted metabolomics combined with much more automated metabolomic analyses have made these techniques much cheaper and more widely accessible. Indeed, the projected cost to quantitatively measure up to 600 metabolites in a drop of blood or urine is predicted to be $<$ USD 30 . These dramatic cost reductions should open the door to widespread adoption and use in precision nutrition applications. As a result, a number of new precision nutrition companies are emerging that are taking advantage of these pricing dynamics. However, a key limitation of metabolomics being applied to diet and nutrition studies is the relatively short-term dietary window (a few hours to a few days) that blood, urine, or stool samples provide. New developments in the area of hair [73-75], fingernail, and toenail metabolomics [76] offer the possibility of analyzing and interpreting much longer term dietary patterns. We expect that, as more metabolomics and nutrition data are collected and more BFIs continue to be identified, it is likely that many more robust connections to health outcomes will become apparent. While this may take some time, these unique datasets and these important health connections will go a long way to making precision nutrition more ubiquitous and more useful to a larger segment of the population.

\section{Conflict of Interest Statement}

The authors have no conflicts of interest to declare.

\section{Funding Sources}

Funding for this work has been provided by Genome Canada, the Canada Foundation for Innovation (CFI), Western Economic Diversification, and the Canadian Institutes of Health Research (CIHR). A.H.K. was funded by postdoctoral fellowships from Alberta Innovates and CIHR.

\section{Author Contributions}

D.S.W. conceived the idea for the manuscript. M.L., A.H.K., and P.Z. performed the literature review and prepared the first draft of the manuscript. D.S.W. edited and revised the final manuscript. All the authors approved the final version of the manuscript.

References

1 Cecil JE, Barton KL. Inter-individual differences in the nutrition response: from research to recommendations. Proc Nutr Soc. 2020 May;79(2):171-3.

2 Andraos S, Wake M, Saffery R, Burgner D, Kussmann M, O’Sullivan J. Perspective: advancing understanding of population nutrient-health relations via metabolomics and precision phenotypes. Adv Nutr. 2019 Nov 1; 10(6):944-52.

3 Wang DD, Hu FB. Precision nutrition for prevention and management of type 2 diabetes. Lancet Diabetes Endocrinol. 2018 May; 6(5):416-26.

4 Rodgers GP, Collins FS. Precision nutritionthe answer to "what to eat to stay healthy". JAMA. 2020 Aug 25;324(8):735-6. 
5 Ramos-Lopez O, Milagro FI, Allayee $\mathrm{H}$, Chmurzynska A, Choi MS, Curi R, et al. Guide for current nutrigenetic, nutrigenomic, and nutriepigenetic approaches for precision nutrition involving the prevention and management of chronic diseases associated with obesity. J Nutrigenet Nutrigenomics. 2017; 10(1-2):43-62.

6 Mullins VA, Bresette W, Johnstone L, Hallmark B, Chilton FH. Genomics in personalized nutrition: can you "eat for your genes"? Nutrients. 2020 Oct 13;12(10):3118.

7 Goldansaz SA, Guo AC, Sajed T, Steele MA, Plastow GS, Wishart DS. Livestock metabolomics and the livestock metabolome: a systematic review. PLoS One. 2017 May 22;12(5): e0177675.

8 Erban A, Fehrle I, Martinez-Seidel F, Brigante F, Más AL, Baroni V, et al. Discovery of food identity markers by metabolomics and machine learning technology. Sci Rep. 2019 Jul 4; 9(1):9697.

9 Scalbert A, Brennan L, Manach C, AndresLacueva C, Dragsted LO, Draper J, et al. The food metabolome: a window over dietary exposure. Am J Clin Nutr. 2014 Jun;99(6):1286308.

10 Varaeva YR, Livantsova EN, Ukrainets IV, Kosyura SD, Starodubova AV. Metabolomics in vitamin status assessment. Curr Pharm Des. 2018;24(26):3028-33.

11 Christopher KB. Nutritional metabolomics in critical illness. Curr Opin Clin Nutr Metab Care. 2018 Mar;21(2):121-5.

12 Gibbons H, Brennan L. Metabolomics as a tool in the identification of dietary biomarkers. Proc Nutr Soc. 2017 Feb;76(1):42-53.

13 Guasch-Ferre M, Bhupathiraju SN, Hu FB. Use of metabolomics in improving assessment of dietary intake. Clin Chem. 2018 Jan; 64(1):82-98.

14 Gibbons H, O'Gorman A, Brennan L. Metabolomics as a tool in nutritional research. Curr Opin Lipidol. 2015 Feb;26(1):30-4.

15 Emwas AH, Roy R, McKay RT, Tenori L, Saccenti E, Gowda GAN, et al. Nmr spectroscopy for metabolomics research. Metabolites. 2019 Jun 27;9(7):123.

16 Emwas AH, Al-Talla ZA, Kharbatia NM. Sample collection and preparation of biofluids and extracts for gas chromatography-mass spectrometry. Methods Mol Biol. 2015;1277: 75-90.

17 Foroutan A, Guo AC, Vazquez-Fresno R, Lipfert M, Zhang L, Zheng J, et al. Chemical composition of commercial cow's milk. J Agric Food Chem. 2019 May 1;67(17):4897-914.

18 Karu N, Deng L, Slae M, Guo AC, Sajed T, Huynh $\mathrm{H}$, et al. A review on human fecal metabolomics: methods, applications and the human fecal metabolome database. Anal Chim Acta. 2018 Nov 7;1030:1-24.

19 Wishart DS. Metabolomics for investigating physiological and pathophysiological processes. Physiol Rev. 2019;99(4):1819-75.
20 Senyuva HZ, Gökmen V, Sarikaya EA. Future perspectives in OrbitrapTM-high-resolution mass spectrometry in food analysis: a review. Food Addit Contam Part A Chem Anal Control Expo Risk Assess. 2015;32(10):1568-606.

21 Bayram M, Gökırmaklı Ç. Horizon scanning: how will metabolomics applications transform food science, bioengineering, and medical innovation in the current era of foodomics? OMICS. 2018 Mar;22(3):177-83.

22 Collins C, McNamara AE, Brennan L. Role of metabolomics in identification of biomarkers related to food intake. Proc Nutr Soc. 2019 May;78(2):189-96.

23 Wild J, Shanmuganathan M, Hayashi M, Potter M, Britz-Mckibbin P. Metabolomics for improved treatment monitoring of phenylketonuria: urinary biomarkers for non-invasive assessment of dietary adherence and nutritional deficiencies. Analyst. 2019 Nov 4; 144(22):6595-608.

24 Neveu V, Moussy A, Rouaix H, Wedekind R, Pon A, Knox C, et al. Exposome-Explorer: a manually-curated database on biomarkers of exposure to dietary and environmental factors. Nucleic Acids Res. 2017 Jan 1;45(D1): D979-84.

25 Gibbons H, Michielsen CJR, Rundle M, Frost G, McNulty BA, Nugent AP, et al. Demonstration of the utility of biomarkers for dietary intake assessment; proline betaine as an example. Mol Nutr Food Res. 2017 Oct;61(10): 1700037.

26 McNamara AE, Walton J, Flynn A, Nugent AP, McNulty BA, Brennan L. The potential of multi-biomarker panels in nutrition research: total fruit intake as an example. Front Nutr. 2020 Jan 14;7:577720.

27 Marsden D, Larson C, Levy HL. Newborn screening for metabolic disorders. J Pediatr. 2006 May 1;148(5):577-84.

28 McHugh D, Cameron CA, Abdenur JE, Abdulrahman M, Adair O, Al Nuaimi SA, et al. Clinical validation of cutoff target ranges in newborn screening of metabolic disorders by tandem mass spectrometry: a worldwide collaborative project. Genet Med. 2011 Mar; 13(3):230-54

29 Blackburn PR, Gass JM, Vairo FPE, Farnham KM, Atwal HK, Macklin S, et al. Maple syrup urine disease: mechanisms and management. Appl Clin Genet. 2017 Sep 6;10:57-66.

30 Korein J, Sansaricq C, Kalmijn M, Honig J, Lange B. Maple syrup urine disease: clinical, EEG, and plasma amino acid correlations with a theoretical mechanism of acute neurotoxicity. Int J Neurosci. 1994;79(1-2):21-45.

31 Vockley J, Andersson HC, Antshel KM, Braverman NE, Burton BK, Frazier DM, et al. Phenylalanine hydroxylase deficiency: diagnosis and management guideline. Genet Med. 2014;16(2):188-200

32 van Spronsen FJ, Hoeksma M, Reijngoud DJ. Brain dysfunction in phenylketonuria: is phenylalanine toxicity the only possible cause? J Inherit Metab Dis. 2009 Feb;32(1):46-51.
33 Canda E, Kalkan Uçar S, Çoker M. Biotinidase deficiency: prevalence, impact and management strategies. Pediatric Health Med Ther. 2020;11:127-33.

34 Wolf B. Biotinidase deficiency: "if you have to have an inherited metabolic disease, this is the one to have". Genet Med.. 2012 Jun;14(6): 565-75.

35 Wishart DS. Metabolomics: applications to food science and nutrition research. Trends Food Sci Technol. 2008 Sep;19(9):482-93.

36 Cory H, Passarelli S, Szeto J, Tamez M, Mattei J. The role of polyphenols in human health and food systems: a mini-review. Front Nutr. 2018 Sep 21;5:87.

37 Oliveira JR, Ribeiro GHM, Rezende LF, Fraga-Silva RA. Plant terpenes on treating cardiovascular and metabolic disease: a review. Protein Pept Lett. 2021 Jan 28.

38 Muroya S, Ueda S, Komatsu T, Miyakawa T, Ertbjerg P. Meatabolomics: muscle and meat metabolomics in domestic animals. Metabolites. 2020 May 11;10(5):188.

39 Yuan Y, Zhao Y, Yang J, Jiang Y, Lu F, Jia Y, et al. Metabolomic analyses of banana during postharvest senescence by $1 \mathrm{H}$-high resolution-NMR. Food Chem. 2017 Mar 1;218:40612.

40 Diamantidou D, Zotou A, Theodoridis G. Wine and grape marc spirits metabolomics. Metabolomics. 2018 Dec 11;14(12):159.

41 Martins C, Brandão T, Almeida A, Rocha SM. Enlarging knowledge on lager beer volatile metabolites using multidimensional gas chromatography. Foods. 2020 Sep 1;9(9):1276.

42 Wang W, Li Y, Dang P, Zhao S, Lai D, Zhou L. Rice secondary metabolites: structures, roles, biosynthesis, and metabolic regulation. Molecules. 2018 Nov 27;23(12):3098.

43 Ara T, Sakurai N, Takahashi S, Waki N, Suganuma $\mathrm{H}$, Aizawa $\mathrm{K}$, et al. TOMATOMET: a metabolome database consists of 7118 accurate mass values detected in mature fruits of 25 tomato cultivars. Plant Direct. 2021 Apr 29;5(4):e00318.

44 Rothwell JA, Perez-Jimenez J, Neveu V, Medina-Remón A, M’hiri N, García-Lobato P, et al. Phenol-explorer 3.0: a major update of the phenol-explorer database to incorporate data on the effects of food processing on polyphenol content. Database. 2013 Oct 7;2013: bat070.

45 PhytoHub [Internet]. [cited 2021 May 15]. Available from: http://phytohub.eu/.

46 FooDB [Internet]. [cited 2021 Apr 30]. Available from: https://foodb.ca/.

47 The FOODBALL Portal [Internet]. [cited 2021 May 15]. Available from: https://foodmetabolome.org/.

48 Brouwer-Brolsma EM, Brennan L, Drevon CA, Van Kranen H, Manach C, Dragsted LO, et al. Combining traditional dietary assessment methods with novel metabolomics techniques: present efforts by the food biomarker alliance. Proc Nutr Soc. 2017 Nov;76(4):61927. 
49 Ulaszewska M, Vázquez-Manjarrez N, Garcia-Aloy M, Llorach R, Mattivi F, Dragsted LO, et al. Food intake biomarkers for apple, pear, and stone fruit. Genes Nutr. 2018 Nov 29;13(1):29.

50 Ulaszewska M, Garcia-Aloy M, VázquezManjarrez N, Soria-Florido MT, Llorach R, Mattivi F, et al. Food intake biomarkers for berries and grapes. Genes Nutr. 2020 Sep 23; 15(1):17.

51 Vázquez-Manjarrez N, Ulaszewska M, Garcia-Aloy M, Mattivi F, Praticò G, Dragsted $\mathrm{LO}$, et al. Biomarkers of intake for tropical fruits. Genes Nutr. 2020 Jun 19;15(1):11.

52 Brouwer-Brolsma EM, Brandl B, Buso MEC, Skurk T, Manach C. Food intake biomarkers for green leafy vegetables, bulb vegetables, and stem vegetables: a review. Genes Nutr. 2020 Apr 9;15(1):7.

53 Gao Q, Praticò G, Scalbert A, Vergères G, Kolehmainen M, Manach C, et al. A scheme for a flexible classification of dietary and health biomarkers. Genes Nutr. 2017 Dec 12; 12(1):34.

54 Dragsted LO, Gao Q, Scalbert A, Vergères G, Kolehmainen M, Manach C, et al. Validation of biomarkers of food intake-critical assessment of candidate biomarkers. Genes Nutr. 2018 May 30;13(1):14.

55 Wishart DS, Feunang YD, Marcu A, Guo AC, Liang K, Vázquez-Fresno R, et al. HMDB 4.0: the human metabolome database for 2018 Nucleic Acids Res. 2018 Jan 1;46(D1):D60817.

56 Wishart DS, Bartok B, Oler E, Liang KYH, Budinski Z, Berjanskii M, et al. MarkerDB: an online database of molecular biomarkers. Nucleic Acids Res. 2021 Jan 8;49(D1):D1259-67.

57 Cross AJ, Major JM, Sinha R. Urinary biomarkers of meat consumption. Cancer Epidemiol Biomarkers Prev. 2011 Jun;20(6):110711.

58 Lloyd AJ, Beckmann M, Favé G, Mathers JC, Draper J. Proline betaine and its biotransformation products in fasting urine samples are potential biomarkers of habitual citrus fruit consumption. Br J Nutr. 2011 Sep;106(6): $812-24$.
59 Gibson R, Lau CE, Loo RL, Ebbels TMD, Chekmeneva E, Dyer AR, et al. The association of fish consumption and its urinary metabolites with cardiovascular risk factors: the International Study of Macro-/Micronutrients and Blood Pressure (INTERMAP). Am J Clin Nutr. 2020 Feb 1;111(2):280-90.

60 Sri Harsha PSC, Wahab RA, Garcia-Aloy M, Madrid-Gambin F, Estruel-Amades S, Watzl $\mathrm{B}$, et al. Biomarkers of legume intake in human intervention and observational studies: a systematic review. Genes Nutr. 2018 Sep 10; 13(1):25.

61 Sri Harsha PSC, Wahab RA, Garcia-Aloy M, Madrid-Gambin F, Estruel-Amades S, Watzl $\mathrm{B}$, et al. Correction to: biomarkers of legume intake in human intervention and observational studies: a systematic review. Genes Nutr. 2018;13:27.

62 Heinzmann SS, Holmes E, Kochhar S, Nicholson JK, Schmitt-Kopplin P. 2-Furoylglycine as a candidate biomarker of coffee consumption. J Agric Food Chem. 2015 Sep 30;63(38): 8615-21.

63 Ordovas JM, Berciano S. Personalized nutrition and healthy aging. Nutr Rev. 2020 Dec 1; 78(12 Suppl 2):58-65.

64 McGarrah RW, Crown SB, Zhang GF, Shah $\mathrm{SH}$, Newgard CB. Cardiovascular metabolomics. Circ Res. 2018;122(9):1238-58.

$65 \mathrm{Au} \mathrm{A}$. Metabolomics and lipidomics of ischemic stroke. Adv Clin Chem. 2018;85:31-69.

66 Arneth B, Arneth R, Shams M. Metabolomics of type 1 and type 2 diabetes. Int J Mol Sci. 2019 May 18;20(10):2467.

67 Centers for Disease Control and Prevention. National Diabetes Statistics Report 2020. Estimates of diabetes and its burden in the United States. 2020.

68 Zeevi D, Korem T, Zmora N, Israeli D, Rothschild D, Weinberger A, et al. Personalized nutrition by prediction of glycemic responses. Cell. 2015 Nov 19;163(5):1079-94.
69 Zmora N, Zeevi D, Korem T, Segal E, Elinav E. Taking it personally: personalized utilization of the human microbiome in health and disease. Cell Host Microbe. 2016 Jan 13;19(1): 12-20.

70 Anwar MA, Barrera-Machuca AA, Calderon N, Wang W, Tausan D, Vayali T, et al. Valuebased healthcare delivery through metabolomics-based personalized health platform. Healthc Manage Forum. 2020 May;33(3): $126-34$.

71 Westerman K, Reaver A, Roy C, Ploch M, Sharoni E, Nogal B, et al. Longitudinal analysis of biomarker data from a personalized nutrition platform in healthy subjects. Sci Rep. 2018 Oct 2;8(1):14685

72 Maruvada P, Lampe JW, Wishart DS, Barupal D, Chester DN, Dodd D, et al. Perspective: dietary biomarkers of intake and exposure-exploration with omics approaches. Adv Nutr. 2020 Mar 1;11(2):200-15.

73 Ehleringer JR, Covarrubias Avalos S, Tipple BJ, Valenzuela LO, Cerling TE. Stable isotopes in hair reveal dietary protein sources with links to socioeconomic status and health. Proc Natl Acad Sci U S A. 2020 Aug 18 117(33):20044-51.

74 Valenzuela LO, O'Grady SP, Enright LE, Murtaugh M, Sweeney C, Ehleringer JR. Evaluation of childhood nutrition by dietary survey and stable isotope analyses of hair and breath. Am J Hum Biol. 2018 May;30(3): e23103.

75 Votruba SB, Shaw PA, Oh EJ, Venti CA, Bonfiglio S, Krakoff J, et al. Associations of plas$\mathrm{ma}, \mathrm{RBCs}$, and hair carbon and nitrogen isotope ratios with fish, meat, and sugar-sweetened beverage intake in a 12-wk inpatient feeding study. Am J Clin Nutr. 2019 Dec 1; 110(6):1306-15.

76 Park K, Seo E. Association between toenai mercury and metabolic syndrome is modified by selenium. Nutrients. 2016 Jul 12;8(7):424. 